\title{
SUSTENTABILIDADE HIDROAMBIENTAL DE NASCENTES NA BACIA HIDROGRÁFICA DO RIO GRAMAME NO ESTADO DA PARAÍBA, BRASIL
}

\section{Hydro-Environmental Sustainability of the Gramame river basin headwaters in Paraiba State, Brazil}

Eudes Oliveira Bomfim

Universidade Federal da Paraíba, João Pessoa, Paraíba, Brasil eudes.bomfim1997@gmail.com

Carmem Lúcia Moreira Gadelha

Universidade Federal da Paraíba, João Pessoa, Paraíba, Brasil carmemgadelha@yahoo.com.br

Hamilcar José Almeida Filgueira Universidade Federal da Paraíba, João Pessoa, Paraíba, Brasil hfilgueira@gmail.com

Jamille Freire Amorim Universidade Federal da Paraíba, João Pessoa, Paraíba, Brasil jamille.fa@gmail.com

Diego da Silva Amorim Universidade Federal da Paraíba, João Pessoa, Paraíba, Brasil diegodsamorim@gmail.com

Artigo recebido em 24/06/2014 e aceito para publicação em 14/11/2015

RESUMO: Este estudo objetivou determinar o nível da sustentabilidade hidroambiental de áreas circunvizinhas às nascentes situadas na bacia hidrográfica do rio Gramame, município de Pedras de Fogo/PB. Foram escolhidas quatro nascentes para o desenvolvimento desta pesquisa: Cacimba da Rosa e Cabelão, na zona periurbana; Nova Aurora e Fazendinha, na zona rural. A metodologia utilizada para obtenção do índice de sustentabilidade foi baseada nos trabalhos de Calório (1997) e Daniel (2001). Elaborou-se um quadro de indicadores para avaliação da sustentabilidade hidroambiental das áreas estudadas. Foram atribuídos pesos de 0 a 1 aos resultados obtidos para cada indicador. Utilizou-se o gráfico tipo radar, pelo qual foi possível comparar de forma dinâmica todas as variáveis pesquisadas. A maior área do triângulo formado no gráfico representa a maior sustentabilidade do indicador. Os índices finais revelaram que a área em torno da nascente Nova Aurora obteve os melhores desempenhos de seus indicadores.

Palavras-Chaves: Sustentabilidade hidroambiental; Nascentes; Bacia hidrográfica; Estado da Paraíba.

ABSTRACT: This study has aimed to determine the level of hydro-environmental sustainability of areas surrounding headwaters in the Gramame river basin, located in Pedras de Fogo county, Paraíba State. Four headwaters 
were chosen for the development of this research: Cacimba da Rosa and Cabelão, in the intermediate rural-urban zone; Nova Aurora and Fazendinha, in the countryside. The methodology used to obtain the sustainability index was based on the work of the Calorie (1997) and Daniel (2001). It was used the radar chart type, by which it was possible to dynamically compare all variables. The largest area of the triangle formed on the chart, is the largest sustainability indicator. The final indices revealed that the area around the Nova Aurora headwaters got the best performances of their indicators.

Keywords: Hydro-environmental sustainability; Headwaters; River basin; Paraíba State.

\section{INTRODUÇÃO}

O entendimento do fenômeno da ocorrência e da degradação de nascentes de rios que abastecem as zonas rurais e urbanas envolve conhecimentos das áreas de geologia, geomorfologia, hidrologia, solos e vegetação, o que justifica as diversas definições e os tipos de surgência. Conforme Cabral da Silva et al. (2011) alguns pesquisadores consideram-nas como do tipo pontual, distribuídas ou difusas, outras apresentam um enfoque puramente hidrológico.

Fatores como a supressão da vegetação, as atividades agropecuárias e o uso inadequado do solo potencializam o processo de degradação de nascentes e dos rios e riachos, propriamente ditos, e interferem na qualidade e quantidade da água de uma bacia hidrográfica. Também, a inaptidão do ambiente, a compactação e o preparo impróprio do solo, a monocultura, a irrigação inadequada, o superpastejo e a cobertura de solo insuficiente degradam áreas de nascentes (KOBIYAMA et al., 2008).

O Relatório de Desenvolvimento Humano elaborado pelo Programa das Nações Unidas para o Desenvolvimento (PNUD) em 2006 destacava que, em diversas partes do mundo, consideráveis reservas de água doce estão sendo degradadas (PNUD, 2006). O desmatamento, a retirada de matas ciliares, a prática de agricultura irrigada com o uso de agrotóxico e a pecuária sem apoio técnico, aliados à expansão da zona urbana, os resíduos doméstico e industrial lançados de forma inadequada no meio ambiente foram apontados como causas principais dessa degradação.

Esse cenário hidroambiental de crescente escassez no suprimento de água de qualidade satisfatória e em quantidade adequada requer dos planejadores sociais e tomadores de decisão a busca por estratégias, em especial da gestão dos usos da água para o consumo humano, de modo a não provocar problemas de disponibilidade para as futuras gerações. Essas estratégias devem ser pautadas na recuperação e preservação dos corpos hídricos, em especial das nascentes, pois, segundo Tundisi (2003), as ações do homem que interferem nos ciclos naturais e na disponibilidade de água de boa qualidade têm comprometido à sustentabilidade dos recursos hídricos. As nascentes têm sido objeto de estudo de vários pesquisadores no Brasil e em âmbito internacional desde o início do século XXI, como se observa em Soares et al. (2010), Ferreira et al. (2009), Menezes et al. (2009), Ferreira et al. (2011), Cabral da Silva et al. (2011), Koperski et al. (2011), Bruins et al. (2012), Fry et al. (2012), Mora et al. (2013) entre outros.

A ideia de sustentabilidade hidroambiental foi definida por Vieira (1996), como a gestão integrada de recursos hídricos de uma região na abrangência de vários aspectos como o ciclo hidrológico, em suas fases superficial, subterrânea e aérea; os usos múltiplos da água; o inter-relacionamento dos sistemas naturais e sociais; a interdependência dos componentes econômicos, sociais, ambientais e políticas de desenvolvimento que, na contemporaneidade, encontram-se qualificados no desenvolvimento sustentável.

Dessa forma, avaliar níveis de sustentabilidade é fazer um juízo de valor sobre o estado dos atributos do meio (como água, solo e ar) com relação à sua influência ou à sua capacidade de atender às condições necessárias para a vida num determinado espaço e tempo. Para essa avaliação, vários parâmetros e variáveis têm sido apontados na literatura como influentes no desempenho de sistemas hídricos e ambientais - os chamados indicadores (XU et al., 2005; FORTINI et al., 2006; FELD et al., 2007; EEA, 2009; MAGALHÃES JÚNIOR, 2010; SINGH et al., 
2009 e 2010). Segundo Vieira e Studart (2009), não parece haver dificuldade em apontar indicadores, mas sim, em agregá-los em um único parâmetro - índice -, capaz de traduzir numericamente uma situação e indicar, ao tomador de decisão, o sentido da sustentabilidade da região.

Então, o desenvolvimento de índices de sustentabilidade hidroambiental tem recebido atenção crescente, conforme os estudos de Vieira (1996), Guimarães (2008), Martins e Cândido (2008), Vieira e Studart (2009), Magalhães Júnior (2010) e Carvalho e Curi (2013). Além destes, outros estudos sobre indicadores e índices de sustentabilidade foram realizados por cientistas e grupos acadêmicos como Hammond et al. (1995), Lindenmayer et al. (2000), Junk (2000), Swart et al. (2002), Xu et al. (2005), Fortini et al. (2006), Feld et al. (2007), EEA (2009), Singh et al. (2009; 2010), Feld et al. (2009; 2010), Santos (2010) e Castello (2011).

Porém, a metodologia para cálculo de um índice de sustentabilidade desenvolvida por Calório (1997) e aperfeiçoada por Daniel (2001), apresenta de forma simples e resumida, mas, suficiente para representar toda a coleção de indicadores levantados em um sistema hídrico por meio da confecção de gráficos tipo radar. Daniel (2001), concluiu que essa metodologia gera índices que apontam o nível do desempenho da sustentabilidade ambiental e suas tendências, além de orientar a tomada de decisões quanto ao uso e ocupação de áreas agroflorestais e, que os gráficos tipo radar se apresentam como uma boa opção para ilustração didática do framework de indicadores e índices.

De acordo com Callado (2010), o desenvolvimento de métodos que relacionam a construção de frameworks, que são o arcabouço para organizar, escolher e medir indicadores de sustentabilidade, é influenciado pela Comissão de Desenvolvimento Sustentável (CDS) das Nações Unidas e pela Organização para Cooperação e Desenvolvimento Econômico (OECD). Segundo Cândido (2004) e Bellen (2006), dentre as metodologias aceitas em estudos de sustentabilidade ambiental estão três sistemas de indicadores: Barômetro da Sustentabilidade (Barometer of Sustainability), Painel da Sustentabilidade
(Dashboard of Sustainability) e Pegada Ecológica (Ecological Footprint Method).

Outros modelos, como o sugerido pelo World Resources Institute (WRI), sistematizam as informações ambientais para torná-las de fácil compreensão ao público (OECD, 1994). Esses modelos adotam a forma de Pressão-Estado-Resposta que tem como objetivos apresentar as questões ambientais de forma que respondam as seguintes indagações: i) o que está acontecendo com o meio ambiente e com a base de recursos naturais?; ii) por que está acontecendo?; e iii) o que está sendo feito para reverter as pressões?

Neste contexto e, considerando a importância de nascentes na formação de rios e riachos, este estudo objetivou a construção de indicadores, apresentados em dimensões (Social, Econômica, Ambiental e Institucional), para determinar o índice de sustentabilidade hidroambiental de áreas em torno de nascentes da bacia hidrográfica do rio Gramame, estado da Paraíba, Nordeste do Brasil. Esta bacia hidrográfica é responsável por cerca de $70 \%$ do abastecimento de água da Grande João Pessoa, que compreende os municípios de João Pessoa (capital do Estado), Cabedelo, Bayeux e parte de Santa Rita e das cidades de Pedras de Fogo e Conde (PDRH, 2001), além de várias comunidades rurais ribeirinhas.

\section{ÁREA DE ESTUDO}

A bacia hidrográfica do rio Gramame (BHRG) possui uma área de drenagem de, aproximadamente, $589,1 \mathrm{~km}^{2}$ e está localizada entre as latitudes $7^{\circ} 11^{\prime}$ e $7^{\circ} 23^{\prime} \mathrm{Sul}$ e as longitudes $34^{\circ} 48^{\prime}$ e $35^{\circ} 10^{\prime}$ 'Oeste, na região litorânea sul do estado da Paraíba, região Nordeste do Brasil. Abrange parte dos municípios de Alhandra, Cruz do Espírito Santo, João Pessoa, Santa Rita, São Miguel de Taipu, Conde e Pedras de Fogo, estes dois últimos com sedes administrativas dentro do espaço territorial da bacia hidrográfica. $\mathrm{O}$ principal curso d'água é o rio Gramame, com extensão de 54,3 km, cujos afluentes mais importantes são os rios Mumbaba, Mamuaba e Água Boa. A alteração do regime de fluxo da água devido ao uso crescente e sem controle do solo para a agricultura é identificada como uma das principais causas da degradação 
ambiental na bacia hidrográfica.

Registram-se conflitos entre a atividade de pesca e a industrial nessa região em decorrência da qualidade da água. Com relação à disponibilidade hídrica destacam-se, entre os conflitos mais notáveis, os que envolvem a demanda de água para a irrigação e o abastecimento humano. Tudo isso ocorre por causa da ocorrência de múltiplos usos de água, como agricultura irrigada, indústria, lazer, piscicultura, dessedentação de animais e abastecimento público (PARAÍBA, 2000). Além do mais, de acordo com Coelho (2011), cerca de $50 \%$ da área da bacia hidrográfica do rio Gramame são ocupadas por culturas da cana-de-açúcar e abacaxi onde são aplicados, de forma inadequada e/ou indiscriminada, fertilizantes sintéticos e agrotóxicos (inseticidas, fungicidas e herbicidas). Dessa forma, nascentes de importantes rios como o Gramame, estão em estado de degradação crescente pela ação antrópica.

A área de estudo localiza-se no alto curso da bacia hidrográfica do rio Gramame, no município de Pedras de Fogo (Figura 1), e envolve quatro (04) importantes nascentes do rio Gramame: Cabelão $(9.181 .918 \mathrm{~m} \mathrm{~N}$ e $266.560 \mathrm{~m} \mathrm{E}$, em coordenadas UTM, SAD 69, Zona 25, Hemisfério Sul), Cacimba da Rosa (9.181.542m N e 265.632m E), Nova Aurora (9.182.495m N e $263.233 \mathrm{~m} \mathrm{E})$ e Fazendinha (9.192.080m N e $262.473 \mathrm{~m} \mathrm{E})$.

Figura 1 - Localização das nascentes no alto curso da bacia hidrográfica do rio Gramame, munícipio de Pedras de Fogo/PB

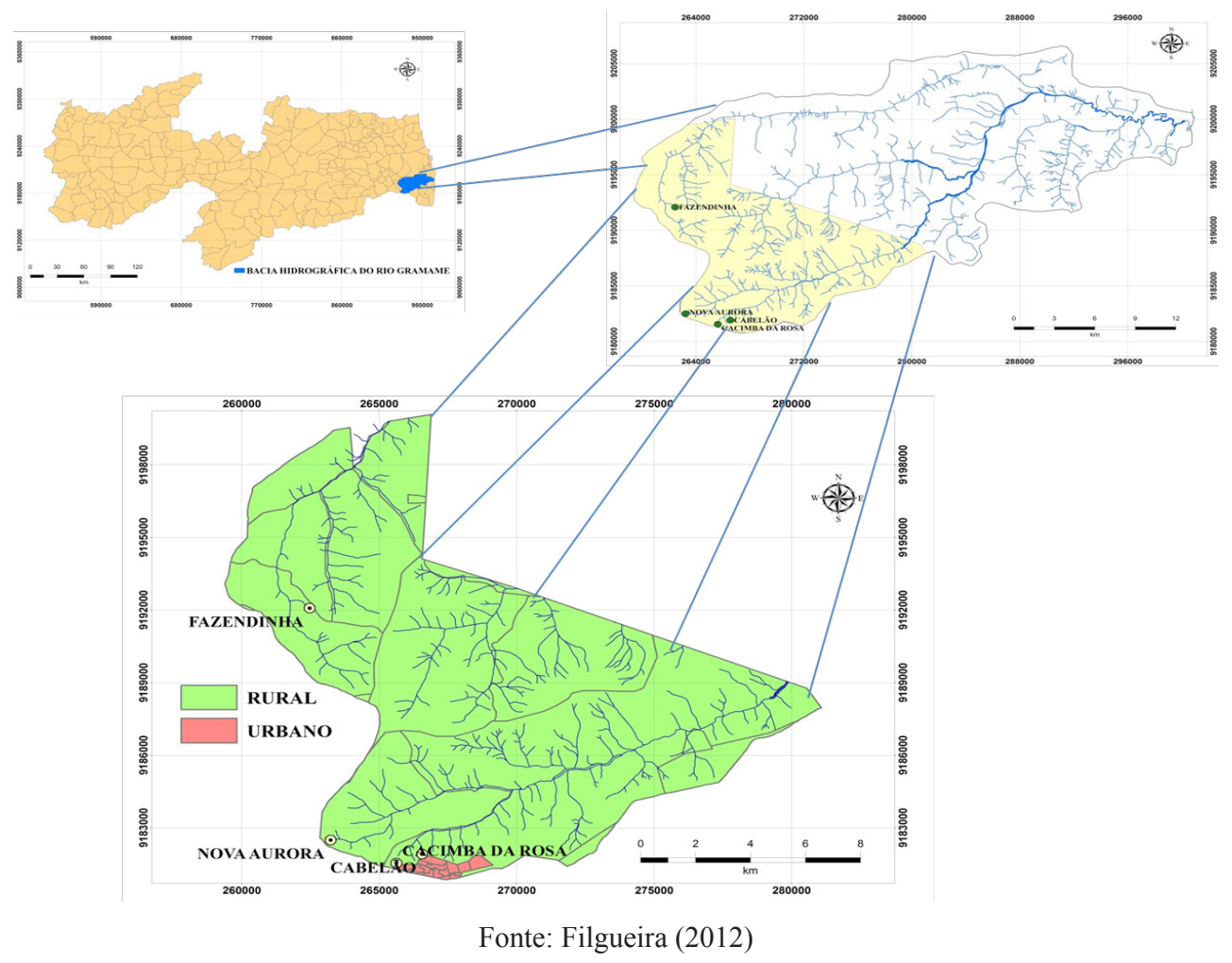

Estas nascentes foram selecionadas por serem de fácil acesso e representativas para a formação do rio Gramame, sendo duas situadas em áreas periurbanas, onde as atividades urbanas e rurais se misturam (Cabelão e Cacimba da Rosa) e duas localizadas na zona rural, em assentamentos agrícolas do Instituto Nacional de Colonização e Reforma Agrária/Incra
(Nova Aurora e Fazendinha).

\section{MATERIAL E MÉTODOS}

Os pilares metodológicos para atingir os objetivos deste estudo foram de caráter exploratório e descritivo. Assim, como dados primários, foram 
levantadas as características e as experiências da população que utiliza a água produzida pelas nascentes, por meio de incursões ao campo e aplicação de questionário semi estruturado. A análise dos dados obtidos teve abordagem quali-quantitativa.

Os parâmetros de qualidade da água cor, cloretos, nitrato, amônia, DBO e coliformes termotolerantes, foram analisados nos laboratórios da Universidade Federal da Paraíba (UFPB), conforme métodos especificados em Standard Methods for the Examination of Waterand Waste Water, editado pela American Public Health Association APHA et al. (2012).

Dados secundários, referentes aos setores censitários do município de Pedras de Fogo/PB, foram extraídos do banco de informações, disponibilizado pelo Instituto Brasileiro de Geografia e Estatística IBGE (IBGE, 2011) e tabulados em uma planilha do Excel.

Considerando-se a localização das nascentes verificou-se que um ou mais setores censitários do município exerciam influência direta sobre elas. Dessa forma, extraíram-se as informações da base de dados do IBGE (2011) que serviram para a análise do cenário da sustentabilidade em cada uma das nascentes.

Os dados primários obtidos, os resultados das análises de qualidade da água, as informações disponibilizadas nos setores censitários (IGBE, 2011) e os resultados do diagnóstico ambiental (FILGUEIRA, 2012) foram utilizados para construção do quadro com 20 indicadores para compor o Índice de Sustentabilidade Hidroambiental (ISH) de áreas em torno das nascentes Cabelão, Cacimba da Rosa, Nova Aurora e Fazendinha, conforme o Quadro 1. Do total de 20 indicadores, 5 compõem a dimensão social, 3 a econômica, 8 a ambiental e 4 a institucional.

Quadro 1- Composição do quadro de indicadores para avaliação da sustentabilidade hidroambiental de nascentes na bacia hidrográfica do Rio Gramame

\begin{tabular}{|c|c|c|}
\hline CÓDIGO & İNDICE/TEMA & INDICADOR \\
\hline \multicolumn{3}{|c|}{$\begin{array}{ll}\text { INDICADORES SOCIAIS - IDS } \\
\text { Densidade populacional }\end{array}$} \\
\hline IDS-02 & & \\
\hline IDS-03 & SAUDE & Doencas de veiculacão hídrica \\
\hline IDS-04 & EDUCAÇÄO & Escolaridade \\
\hline IDS-05 & HABITAÇÃO & $\begin{array}{l}\text { Densidade domiciliar } \\
\text { NÔMICOS - IDE }\end{array}$ \\
\hline IDE-01 & INDICADORES ECONÔMICOS - IDE & Renda familiar \\
\hline IDE-02 & PRODUÇÄO AGRİCOLA & Produção \\
\hline IDE-03 & CREDITO & Acesso a crédito \\
\hline & INDICADORES AMBIENTAIS - IDA & $\begin{array}{l}\text { BIENIAIS - IDA } \\
\text { Vazão das nascentes }\end{array}$ \\
\hline $\begin{array}{l}\text { IDA 01 } \\
\text { IDA-02 }\end{array}$ & PRODUÇÃO E QUALIDADE DE ÁGUA & $\begin{array}{l}\text { Qualidade de água (Coliformes, Cor, Cloretos, Nitrato, } \\
\text { Amônia e DBO) }\end{array}$ \\
\hline IDA-03 & CHUVAS & Precipitação \\
\hline I D A - 04 & \multirow{3}{*}{ SANEAMENTO } & Coleta de resíduos \\
\hline IDA-05 & & Rede de esgoto \\
\hline IDA-06 & & Abastecimento de água \\
\hline IDA-07 & DEFENSIVO AGRÍCOLA & Uso de defensivo agrícola \\
\hline IDA-08 & VEGETAÇÃO & Flora nativa \\
\hline \multicolumn{3}{|c|}{ INDICADORES INSTITUCIONAIS - IDI } \\
\hline IDI-01 & \multirow{5}{*}{ PARTICIPAÇÃO INSTITUCIONAL } & Participação cooperativa, associação, sindicato... \\
\hline IDI-02 & & Execução de Projetos \\
\hline IDI-03 & & Capacitação/treinamento \\
\hline IDI-04 & & Monitoramento das áreas \\
\hline TOTAL DE I & & 20 \\
\hline
\end{tabular}


Considerando-se o método desenvolvido por Calório (1997) e aperfeiçoado por Daniel (2001), citados no referencial teórico e recomendados pelos organismos internacionais e nacionais apresenta-se, a seguir, a composição do sistema de indicadores para avaliação da sustentabilidade hidroambiental de áreas em torno de nascentes da bacia hidrográfica do rio Gramame/PB.

De posse dos indicadores e subindicadores por dimensões, foram atribuídos pesos com variação entre 0 e 1 , conforme o nível de desempenho de cada um. Dessa forma, quanto mais próximo do peso igual a 1, mais sustentável o indicador em sua dimensão. Segundo Magalhães Júnior (2010), a atribuição de pesos pode seguir vários critérios e técnicas estatísticas, contudo, nem sempre é possível a utilização dessa recomendação sem que se incorporem níveis de subjetividade na avaliação. Têm-se como exemplo os parâmetros de qualidade da água que, quanto mais distantes dos padrões recomendados pela legislação receberam pesos próximos de 0 a 0,25 . A Tabela 1 ilustra o corolário atribuído para todos os indicadores das dimensões analisados.

Tabela 1- Pesos e corolário do desempenho do indicador

PESO/NÍVEL DESEMPENHO

\begin{tabular}{ll}
\hline $0 \leq$ IS $\leq 0,25$ & INSUSTENTÁVEL \\
$0,25 \leq$ IS $\leq 0,50$ & INTERMEDIÁRIO \\
$0,50 \leq$ IS $\leq 0,75$ & QUASE SUSTENTÁVEL \\
$0,75 \leq$ IS $\leq 1$ & SUSTENTÁVEL \\
\hline
\end{tabular}

Fonte: Elaboração dos Autores

A determinação do Índice de Sustentabilidade Hidroambiental (ISH) proposto para as nascentes seguiu uma sequência de cálculo, utilizando-se de equações matemáticas, distribuídas em seis passos como descritos:

\section{$\checkmark \quad$ 1ㅇ Passo}

Inicialmente, foi necessário padronizar todas as dimensões dos indicadores. Esse procedimento foi realizado para eliminar os efeitos de escalas e de unidades de medida, uma vez que elas se referem a indicadores diferentes. Esse procedimento assegurou que cada variável empregada no cálculo do ISH tivesse o mesmo peso relativo. Para tanto, os dados foram lançados na planilha eletrônica que calculou a média e o desvio padrão das dimensões (social, econômica, ambiental e institucional) de todos os indicadores, utilizando-se a Equação 1.

$$
\mathrm{p}_{\mathrm{n}}=\frac{5+\left(\mathrm{x}_{\mathrm{n}}-\overline{\mathrm{x}}\right)}{\mathrm{S}}
$$

Onde:

$\mathrm{vp}_{\mathrm{n}}$ : valor padronizado do indicador;

$\mathrm{x}_{\mathrm{n}}$ : dimensão real do indicador;

$\overline{\mathrm{x}}$ : valor médio de todos os indicadores;

S: desvio-padrão de todos os indicadores;

$5=$ constante para eliminar valores negativos ou iguais a zero.



Nessa etapa calcula-se o ângulo formado por dois indicadores adjacentes. Utiliza-se para tanto, a Equação trigonométrica 2. 


$$
\alpha=\frac{360}{\mathrm{~N}} \cdot \frac{\pi}{180}
$$

Onde:

$\alpha$ : ângulo formado entre os eixos do gráfico radar, em radianos;

$\mathrm{N}$ : número de indicadores estudados;

$\pi$ : valor de pi, adimensional.

\section{3으 Passo}

De acordo com Daniel (2001), é necessário, também, conhecer as áreas dos triângulos formados no gráfico tipo radar. Dois lados do triângulo têm medidas iguais à dimensão padronizada de indicadores adjacentes. Porém, é preciso determinar o terceiro lado para que seja possível o cálculo da área do triângulo. Esse lado desconhecido é calculado a partir da Equação 3.

$$
\mathrm{d}_{\mathrm{n}}=\sqrt{\left(\mathrm{p}_{\mathrm{n}}\right)^{2}+\left(\mathrm{p}_{\mathrm{n}}+1\right)^{2}-2\left(\mathrm{p}_{\mathrm{n}} \cdot \mathrm{p}_{\mathrm{n}+1}\right) \cos \alpha}
$$

Onde:

$\mathrm{d}_{\mathrm{n}}$ : lado desconhecido do triângulo;

$\mathrm{vp}_{\mathrm{n}}$ : dimensão padronizado do indicador $\mathrm{n}$;

$\mathrm{n}+1$;

$\mathrm{vp}_{\mathrm{n}}+1$ : dimensão padronizado do indicador

$\cos \alpha=\operatorname{cosseno}$ do ângulo $\alpha$ formado entre os eixos.

\section{$\checkmark \quad 4^{\circ}$ Passo}

Após conhecer os três lados do triangulo calcula-se o semiperímetro que serve de ferramenta para o cálculo da área. O semiperímetro é obtido a partir da Equação 4.

$$
P_{n}=\frac{P_{n}+P_{n+1}+d_{n}}{2}
$$

Onde:

$\mathrm{P}_{\mathrm{n}}$ : semiperímetro do triângulo;

$\mathrm{vp}_{\mathrm{n}}$ : dimensão padronizada do indicador $\mathrm{n}$;

$\mathrm{vp}_{\mathrm{n}}+1$ : dimensão padronizada do indicador

$\mathrm{n}+1$;

$\mathrm{d}_{\mathrm{n}}$ : lado desconhecido do triângulo.

\section{5o Passo}

Após todos os cálculos, a área do triângulo é, então, calculada conforme a Equação 5. Esse procedimento é executado para todos os triângulos/ dimensões.

$$
\mathrm{S}_{\mathrm{n}}=\sqrt{\mathrm{P}_{\mathrm{n}}}\left(\mathrm{P}_{\mathrm{n}}-\mathrm{P}_{\mathrm{n}}\right) \times\left(\mathrm{P}_{\mathrm{n}}-\mathrm{P}_{\mathrm{n}+1}\right) \times\left(\mathrm{P}_{\mathrm{n}}-\mathrm{d}_{\mathrm{n}}\right)
$$

Onde:

$\mathrm{S}_{\mathrm{n}}$ : área do triângulo;

$\mathrm{p}_{\mathrm{n}}$ : semiperímetro do triângulo;

$\mathrm{vp}_{\mathrm{n}}$ : dimensão padronizada do indicador $\mathrm{n}$;

$\mathrm{vp}_{\mathrm{n}}+1$ : dimensão padronizada do indicador

$\mathrm{n}+1$;

$\mathrm{d}_{\mathrm{n}}$ : lado desconhecido do triângulo.

$\checkmark$ 6o Passo

Ao final, as áreas dos triângulos são somadas para a obtenção do ISH, calculado a partir da Equação 6, a seguir:

$$
\mathrm{ISH}=\sum_{\mathrm{n}=1}^{\mathrm{N}} \mathrm{S}_{\mathrm{n}}
$$

Onde:

ISH: Índice de Sustentabilidade Hidroambiental;

$\mathrm{N}$ : número de indicadores;

Sn: área dos triângulos.

As equações foram programadas em uma planilha eletrônica do Excel para receber as informações e gerar os gráficos tipos radar. Assim, para se obter o ISH somaram-se as áreas de todos os triângulos do gráfico tipo radar de cada área das nascentes estudadas.

\section{ANÁLISE E DISCUSSÃo DOS RESULTADOS}

\section{$\checkmark$ Dimensão Social}

A Tabela 2 apresenta o desempenho dos indicadores que compuseram a dimensão social estudada. Observa-se que, no geral, os indicadores de densidade populacional, de densidade domiciliar e de escolaridade apresentaram os melhores desempenhos nas áreas em torno das nascentes demonstrando uma situação intermediária até uma condição de 
sustentabilidade. Exceção ocorreu para a nascente Cabelão que se apresentou insustentável no quesito densidade populacional. Já o indicador doenças de veiculação hídrica apresentou uma condição de insustentabilidade, nas nascentes Cacimba da Rosa e Cabelão até a sustentabilidade em Nova Aurora.
Contudo, constatou-se que o indicador Hospitais/ PSF (Programa de Saúde da Família) apresentou o pior desempenho, para todas as áreas deste estudo, o que interfere na qualidade de vida da população envolvida.

Tabela 2- Indicadores da dimensão social e área dos triângulos

\begin{tabular}{|c|c|c|c|c|c|}
\hline \multirow{2}{*}{\multicolumn{2}{|c|}{ INDICADORES SOCIAIS }} & \multicolumn{4}{|l|}{ NASCENTES } \\
\hline & & Cacimba da Rosa & Cabelão & Nova Aurora & Fazendinha \\
\hline \multicolumn{2}{|c|}{ Densidade populacional } & \multirow{2}{*}{$\begin{array}{l}160,242 \\
161.849\end{array}$} & 159,439 & 161,849 & 161,849 \\
\hline \multicolumn{2}{|c|}{ Densidade domiciliar } & & 161,849 & 161,045 & 161,045 \\
\hline \multicolumn{2}{|c|}{ Escolaridade } & 161,045 & 161,849 & 161,045 & 160,242 \\
\hline \multicolumn{2}{|c|}{ Doenças veiculação hídrica } & 159,439 & 159,439 & 161,849 & 161,045 \\
\hline \multicolumn{2}{|c|}{ Hospitais/PSF } & 159,439 & 159,439 & 159,439 & 159,439 \\
\hline \multicolumn{2}{|c|}{ Média do Índice } & 160,402 & 160,403 & 161,045 & 160,724 \\
\hline \multicolumn{2}{|c|}{$\sum$ Área dos triângulos } & 34774,893 & 34774,893 & 35054,054 & 34913,538 \\
\hline Legenda: & SUSTENTÁVEL & $\begin{array}{l}\text { QUASE } \\
\text { SUSTENTÁVE }\end{array}$ & INTERI & DIÁRIO & NTÁVEL \\
\hline
\end{tabular}

Fonte: Elaboração dos autores

Pelo resultado do cálculo da média simples envolvendo todos os indicadores da dimensão social e também pelo maior valor obtido para a área do triângulo, pode-se inferir que a nascente Nova Aurora é quase sustentável para essa dimensão.

\section{$\checkmark$ Dimensão Econômica}

Com relação à dimensão econômica, verificase pela Tabela 3 que os seus três indicadores: renda familiar, acesso a crédito e produção apresentaram desempenho satisfatório em, praticamente, todas as nascentes, caracterizando uma condição intermediária até a sustentabilidade. Apenas o indicador renda familiar da área da nascente Fazendinha apresentouse como insustentável.

Tabela 3- Índices e área dos triângulos da dimensão econômica

\begin{tabular}{l|llll}
\hline \multirow{2}{*}{ INDICADORES ECONÔMICOS } & NASCENTES & & & \\
\cline { 2 - 5 } & Cacimba da Rosa & Cabelão & Nova Aurora & Fazendinha \\
\hline Renda Familiar & 138,835 & 138,159 & 139,547 & 136,771 \\
Acesso a crédito & 139,547 & 139,547 & 139,547 & 139,547 \\
$\begin{array}{l}\text { Produção } \\
\text { Média do Índice }\end{array}$ & 137,465 & 137,465 & 139,547 & 139,547 \\
\hline \multirow{2}{*}{$\sum$ Área dos triângulos } & 138,615 & 138,390 & 139,547 & 138,621 \\
\hline \multirow{2}{*}{ SUSTENTÁVEL } & 15582,786 & 15530,821 & 15791,819 & 15582,396 \\
& & & & \\
& QUASE & SUSTENTÁVEL & INTERMEDIÁRIO & INSUSTENTÁVEL \\
& Fonte: Elaboração dos autores & & \\
& & &
\end{tabular}


Observando-se o resultado do cálculo da média simples envolvendo todos os indicadores da dimensão social e também o maior valor obtido para a área do triângulo, verifica-se que, também nessa dimensão, a nascente Nova Aurora foi a que apresentou melhor desempenho, ou seja, sustentável.

\section{$\checkmark$ Dimensão Ambiental}

A Tabela 4 ilustra o corolário e os cálculos para todos os indicadores e subindicadores da dimensão ambiental analisados. Observa-se que, o indicador de qualidade da água e seus subindicadores apresentaram desempenho que índica uma situação que variou de intermediária a sustentável, na área das nascentes estudadas, pois, os parâmetros cor, amônia, DBO estiveram abaixo dos limites máximos permitidos pela Resolução do Conselho Nacional de Meio Ambiente (CONAMA) no 357/2005, para águas de classes I e II. Por outro lado, o subindicador nitrato, mostrou baixo desempenho e, por isto, insustentável para a água das nascentes Cacimba da Rosa e Cabelão.

Tabela 4- Indicadores e área dos triângulos da dimensão ambiental

\begin{tabular}{|c|c|c|c|c|c|}
\hline \multirow{2}{*}{\multicolumn{2}{|c|}{$\begin{array}{l}\text { INDICADORES/SUBINDICADORES } \\
\text { AMBIENTAIS }\end{array}$}} & \multicolumn{4}{|l|}{ NASCENTES } \\
\hline & & Cacimba da Rosa & Cabelão & Nova Aurora & Fazendinha \\
\hline \multicolumn{2}{|l|}{ Vazão } & 113,557 & 112,431 & 111,868 & 111,868 \\
\hline \multicolumn{2}{|l|}{ Precipitação } & 113,557 & 113,557 & 113,557 & 113,557 \\
\hline \multicolumn{2}{|l|}{ Vegetação } & 111,305 & 111,305 & 112,431 & 113,557 \\
\hline \multirow{3}{*}{ Saneamento } & Coleta de resíduos & 113,557 & 113,557 & 111,305 & 111,305 \\
\hline & Rede de esgoto & 111,305 & 111,868 & 111,305 & 111,305 \\
\hline & Abastecimento de água & 112,994 & 112,431 & 111,305 & 111,305 \\
\hline \multirow{6}{*}{ Qualidade da água } & Coliformes & 112,994 & 113,557 & 113,557 & 112,994 \\
\hline & Cor & 113,557 & 113,557 & 113,557 & 113,557 \\
\hline & Cloretos & 111,868 & 112,431 & 113,557 & 113,557 \\
\hline & Nitrato & 111,305 & 111,305 & 113,557 & 113,557 \\
\hline & Amônia & 113,557 & 113,557 & 113,557 & 113,557 \\
\hline & DBO & 113,557 & 113,557 & 113,557 & 113,557 \\
\hline \multicolumn{2}{|c|}{ Defensivo agrícola } & 111,305 & 111,305 & 111,305 & 111,305 \\
\hline \multicolumn{2}{|l|}{ Média do Índice } & 112,647 & 112,647 & 112,647 & 112,690 \\
\hline \multicolumn{2}{|c|}{$\sum$ Área dos triângulos } & 25518,934 & 25534,522 & 25677,708 & 25646,064 \\
\hline
\end{tabular}

Legenda:

$$
\text { SUSTENTÁVEL }
$$

\section{QUASE}

Em termos de produção de água, a nascente Cacimba da Rosa é sustentável, pois, apresenta uma maior vazão. As demais têm uma sustentabilidade intermediária. Com relação à precipitação, verificase que todas as áreas apresentaram sustentabilidade no desempenho deste indicador.

Quanto ao saneamento, os indicadores e subindicadores apresentaram problemas relacionados a sustentabilidade ambiental nas áreas das nascentes Nova Aurora e Fazendinha, visto que não há infraestrutura local para os serviços de coleta de resíduos sólidos, de esgotamento sanitário e de abastecimento de água. Por outro lado, para as áreas em torno das nascentes periurbanas Cacimba da Rosa 
e Cabelão o subindicador coleta de resíduos sólidos apresentou-se como sustentável e atende ao setor censitário analisado. Já os outros subindicadores abastecimento de água e esgotamento sanitário, o setor de influência a essas nascentes revelaramse, em geral, variando de quase sustentável a uma situação intermediária, menos para o serviço de esgotamento sanitário na área de abrangência da nascente Cacimba da Rosa que apresentou uma situação de insustentabilidade.

Com relação ao indicador vegetação, a flora nativa se faz bem presente na área do entorno da nascente Fazendinha, estando, também, em melhor estado de conservação de exemplares florestais. Contudo, as áreas das nascentes Cacimba da Rosa, Cabelão e NovaAurora apresentaram sustentabilidade intermediária, haja vista à degradação ambiental observada, decorrente de práticas agrícolas incontroladas e da supressão de exemplares da flora local.

\section{$\checkmark$ Dimensão Institucional}

Os indicadores da dimensão institucional apresentaram comportamento igual entre as nascentes estudadas, ou seja, condição de insustentabilidade nas áreas de influencia. Exceção ocorreu, no entanto, para o indicador participação associativa, que revelou bom desempenho em todas as nascentes. A Tabela 5 apresenta o corolário e os índices ponderados, calculados para todos os indicadores da dimensão institucional.

Tabela 5- Índices e área dos triângulos da dimensão institucional

\begin{tabular}{|c|c|c|c|c|}
\hline \multirow{2}{*}{ INDICADORES INSTITUCIONAIS } & \multicolumn{4}{|l|}{ NASCENTES } \\
\hline & Cacimba da Rosa & Cabelão & Nova Aurora & Fazendinha \\
\hline Participação associativa & 155,962 & 155,962 & 155,962 & 155,962 \\
\hline Monitoramento das áreas & 153,383 & 153,383 & 153,383 & 153,383 \\
\hline Capacitação & 153,383 & 153,383 & 153,383 & 153,383 \\
\hline Projetos & 153,383 & 153,383 & 153,383 & 153,383 \\
\hline Média do Índice & 154,027 & 154,027 & 154,027 & 154,027 \\
\hline$\sum$ área dos triângulos & 23614,183 & 23614,183 & 23614,183 & 23614,183 \\
\hline
\end{tabular}

Legenda:

\section{$\begin{array}{ll}\text { SUSTENTÁVEL } & \text { QUASE } \\ \text { SUSTENTÁVEL }\end{array}$}

INTERMEDIÁRIO

INSUSTENTÁVEL

Fonte: Elaboração dos autores

\section{Sustentabilidade Hidroambiental: dos Indicadores aos Índices}

O Gráfico 1 (a,b, c e d) ilustra o somatório dos triângulos de cada área das nascentes pesquisadas na bacia hidrográfica do rio Gramame. Dentre as quatro estudadas, a nascente Nova Aurora, e em seguida Fazendinha, apresentou maior área do triângulo no gráfico de radar, e consequentemente, a maior sustentabilidade hidroambiental. Essa constatação está mais bem visualizada na planilha eletrônica utilizada para processar os dados e informações obtidos (Tabela 6).
As áreas dos triângulos das nascentes Cacimba da Rosa e Cabelão foram menores, o que inferem aos indicadores de sustentabilidade ambiental menores desempenhos. Tal fato pode ser atribuído à ação antrópica marcante nas áreas adjacentes, o que interfere pontualmente nas quatro dimensões pesquisadas. 
Gráfico 1- Somatório dos triângulos de cada área de nascente pesquisada na bacia hidrográfica do rio Gramame: (a) Cacimba da Rosa; (b) Cabelão; (c) Nova Aurora; e (d) Fazendinha
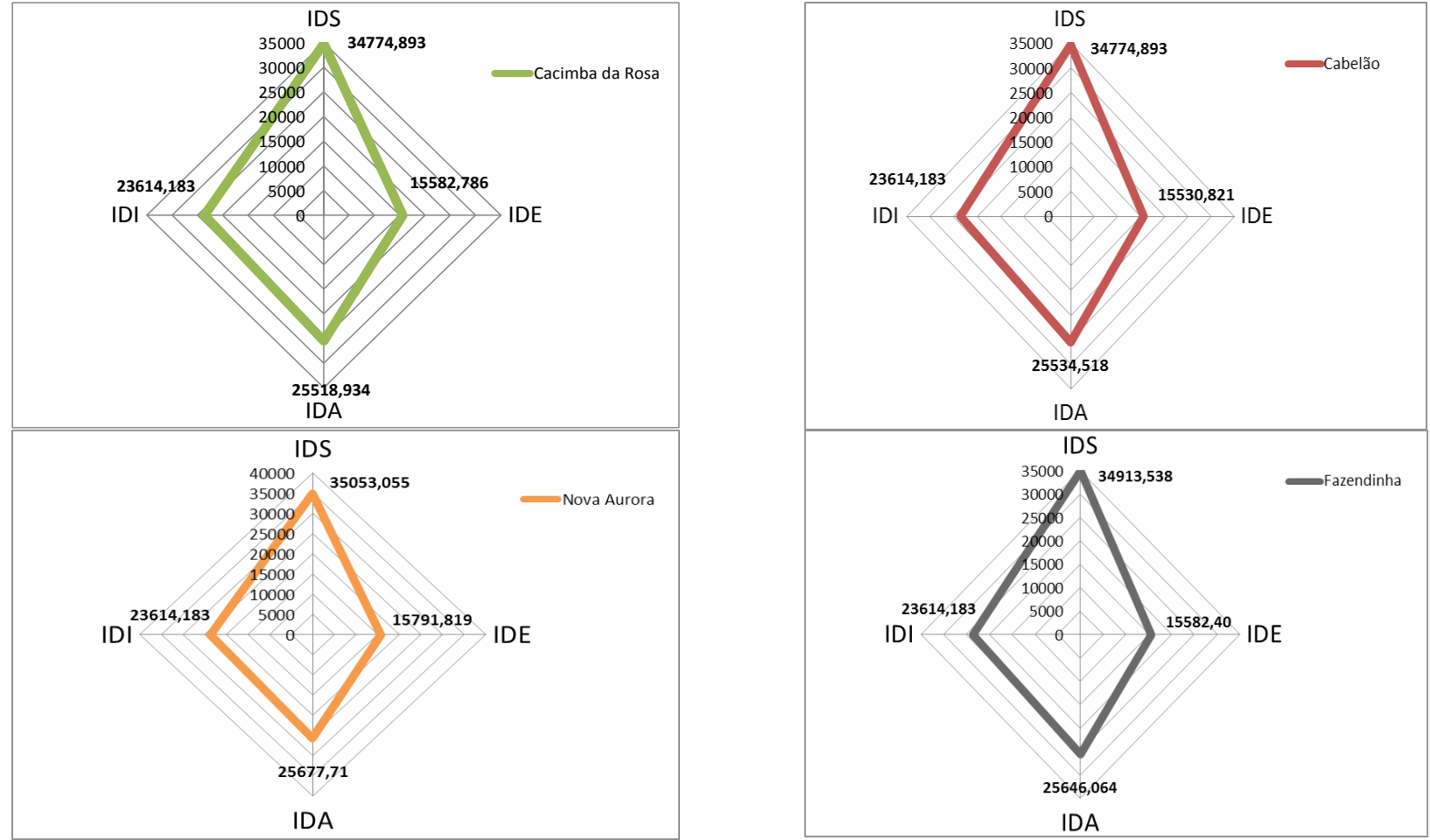

Fonte: Elaboração dos autores

Tabela 6 - Memorial de Cálculo

Cálculo final de indicadores - Dimensão Social (IDS)

\begin{tabular}{|c|c|c|c|c|c|}
\hline CACIMBA DA ROSA & $\begin{array}{l}\text { Densidade } \\
\text { populacional }\end{array}$ & $\begin{array}{l}\text { Hospitais/postos } \\
\text { de saúde }\end{array}$ & $\begin{array}{l}\text { Doenças de } \\
\text { veiculação } \\
\text { hídrica }\end{array}$ & Escolaridade & $\begin{array}{l}\text { Densidade } \\
\text { habitacional }\end{array}$ \\
\hline $\begin{array}{l}\text { DIMENSÃO DO } \\
\text { INDICADOR }\end{array}$ & 0,50 & 0,25 & 0,25 & 0,75 & 1,00 \\
\hline $\begin{array}{l}\text { Valor padronizado do } \\
\text { indicador }\end{array}$ & 160,2422561 & 159,4390368 & 159,4390368 & 161,0454754 & 161,8486948 \\
\hline $\begin{array}{l}\text { Lado desconhecido do } \\
\text { triângulo }\end{array}$ & 90,06534182 & 89,83575786 & 90,30148685 & 90,97045519 & 90,75399433 \\
\hline Semiperímetro do triângulo & 204,8733174 & 204,3569157 & 205,3929995 & 206,9323127 & 206,4224726 \\
\hline Área do triângulo & 6906,200204 & 6871,582659 & 6940,817749 & 7045,716758 & 7010,576026 \\
\hline$\sum$ Áreas & 34774,8934 & & & & \\
\hline CABELÃO & $\begin{array}{l}\text { Densidade } \\
\text { populacional }\end{array}$ & $\begin{array}{l}\text { Hospitais/postos } \\
\text { de saúde }\end{array}$ & $\begin{array}{l}\text { Doenças de } \\
\text { veiculação } \\
\text { hídrica }\end{array}$ & Escolaridade & $\begin{array}{l}\text { Densidade } \\
\text { habitacional }\end{array}$ \\
\hline $\begin{array}{l}\text { DIMENSÃO DO } \\
\text { INDICADOR }\end{array}$ & 0,25 & 0,25 & 0,25 & 1,00 & 1,00 \\
\hline $\begin{array}{l}\text { Valor padronizado do } \\
\text { indicador }\end{array}$ & 159,4390368 & 159,4390368 & 159,4390368 & 161,8486948 & 161,8486948 \\
\hline
\end{tabular}


Sustentabilidade hidroambiental de nascentes na bacia hidrográ ica do rio Gramame no Estado da Paraíba, Brasil Eudes Oliveira Bom im, Carmem Lúcia Moreira Gadelha, Hamilcar José Almeida Filgueira,

Jamille Freire Amorim, Diego da Silva Amorim

\begin{tabular}{|c|c|c|c|c|c|}
\hline $\begin{array}{l}\text { Lado desconhecido do } \\
\text { triângulo }\end{array}$ & 89,83575786 & 89,83575786 & 90,54414161 & 91,19347712 & 90,54414161 \\
\hline Semiperímetro do triângulo & 204,3569157 & 204,3569157 & 205,9159366 & 207,4454333 & 205,9159366 \\
\hline Área do triângulo & 6871,582659 & 6871,582659 & 6975,435294 & 7080,85749 & 6975,435294 \\
\hline$\sum$ Áreas & \multicolumn{5}{|l|}{34774,8934} \\
\hline NOVAAURORA & $\begin{array}{l}\text { Densidade } \\
\text { populacional }\end{array}$ & $\begin{array}{l}\text { Hospitais/postos } \\
\text { de saúde }\end{array}$ & $\begin{array}{l}\text { Doenças de } \\
\text { veiculação } \\
\text { hídrica }\end{array}$ & Escolaridade & $\begin{array}{l}\text { Densidade } \\
\text { habitacional }\end{array}$ \\
\hline $\begin{array}{l}\text { DIMENSÃO DO } \\
\text { INDICADOR }\end{array}$ & 1,00 & 0,25 & 1,00 & 0,75 & 0,75 \\
\hline $\begin{array}{l}\text { Valor padronizado do } \\
\text { indicador }\end{array}$ & 161,8486948 & 159,4390368 & 161,8486948 & 161,0454754 & 161,0454754 \\
\hline $\begin{array}{l}\text { Lado desconhecido do } \\
\text { triângulo }\end{array}$ & 90,54414161 & 90,54414161 & 90,97045519 & 90,74090403 & 90,97045519 \\
\hline Semiperímetro do triângulo & 205,9159366 & 205,9159366 & 206,9323127 & 206,4159275 & 206,9323127 \\
\hline Área do triângulo & 6975,435294 & 6975,435294 & 7045,716758 & 7010,750422 & 7045,716758 \\
\hline$\sum$ Áreas & \multicolumn{5}{|l|}{35053,05453} \\
\hline FAZENDINHA & $\begin{array}{l}\text { Densidade } \\
\text { populacional }\end{array}$ & $\begin{array}{l}\text { Hospitais/postos } \\
\text { de saúde }\end{array}$ & $\begin{array}{l}\text { Doenças de } \\
\text { veiculação } \\
\text { hídrica }\end{array}$ & Escolaridade & $\begin{array}{l}\text { Densidade } \\
\text { habitacional }\end{array}$ \\
\hline $\begin{array}{l}\text { DIMENSÃO DO } \\
\text { INDICADOR }\end{array}$ & 1,00 & 0,25 & 0,75 & 0,50 & 0,75 \\
\hline $\begin{array}{l}\text { Valor padronizado do } \\
\text { indicador }\end{array}$ & 161,8486948 & 159,4390368 & 161,0454754 & 160,2422561 & 161,0454754 \\
\hline $\begin{array}{l}\text { Lado desconhecido do } \\
\text { triângulo }\end{array}$ & 90,54414161 & 90,30148685 & 90,51789842 & 90,51789842 & 90,97045519 \\
\hline Semiperímetro do triângulo & 205,9159366 & 205,3929995 & 205,902815 & 205,902815 & 206,9323127 \\
\hline Área do triângulo & 6975,435294 & 6940,817749 & 6975,784085 & 6975,784085 & 7045,716758 \\
\hline$\sum$ Áreas & \multicolumn{5}{|l|}{34913,53797} \\
\hline
\end{tabular}

Cálculo final de indicadores - Dimensão Econômica (IDE)

\begin{tabular}{|c|c|c|c|}
\hline CACIMBA DA ROSA & Renda familiar & Produção & Acesso a crédito \\
\hline DIMENSÃO DO INDICADOR & 0,75 & 0,25 & 1,00 \\
\hline Valor padronizado do indicador & 138,8534152 & 137,4654593 & 139,5473931 \\
\hline Lado desconhecido do triângulo & 77,85718027 & 78,06686235 & 78,435148 \\
\hline Semiperímetro do triângulo & 177,0880274 & 177,5398574 & 178,4179781 \\
\hline $\begin{array}{l}\text { Área do triângulo } \\
\sum \text { Areas }\end{array}$ & $\frac{5159,618657}{1558278647}$ & 5185,406006 & 5237,761809 \\
\hline CABELÃO & Renda familiar & Produção & Acesso a crédito \\
\hline DIMENSÃO DO INDICADOR & 0.50 & 0.25 & 1.00 \\
\hline Valor padronizado do indicador & 138,1594372 & 137,4654593 & 139,5473931 \\
\hline Lado desconhecido do triângulo & 77,65313421 & 78,06686235 & 78,24814448 \\
\hline Semiperímetro do triângulo & 176,6390154 & 177,5398574 & 177,9774874 \\
\hline Área do triângulo & 5133,831309 & 5185,406006 & 5211,583907 \\
\hline$\sum$ Áreas & 15530,82122 & & \\
\hline NOVA AURORA & Renda familiar & Produção & Acesso a crédito \\
\hline DIMENSÃO DO I] & 15582,39592 & 15582,39592 & 15582,39592 \\
\hline Valor padronizado do indicador & 139,5473931 & 139,5473931 & 139,5473931 \\
\hline
\end{tabular}

Soc. \& Nat., Uberlândia, 27 (3): 453-468, set/dez/2015 


\begin{tabular}{|c|c|c|c|}
\hline Lado desconhecido do triângulo & 78,6278321 & 78,6278321 & 78,6278321 \\
\hline Semiperímetro do triângulo & 178,8613091 & 178,8613091 & 178,8613091 \\
\hline Área do triângulo & 5263,93971 & 5263,93971 & 5263,93971 \\
\hline$\sum$ Areas & 15791,81913 & & \\
\hline FAZENDINHA & Renda familiar & Produção & Acesso a crédito \\
\hline DIMENSÃO DO INDICADOR & 0,00 & 1,00 & 1,00 \\
\hline Valor padronizado do indicador & 136,7714814 & 139,5473931 & 139,5473931 \\
\hline Lado desconhecido do triângulo & 77,89134157 & 78,6278321 & 77,89134157 \\
\hline Semiperímetro do triângulo & 177,105108 & 178,8613091 & 177,105108 \\
\hline Área do triângulo & 5159,228104 & 5263,93971 & 5159,228104 \\
\hline$\sum$ Áreas & 15582,39592 & & \\
\hline
\end{tabular}

O Gráfico 2 mostra o cálculo final de todas as áreas dos triângulos, considerando as quatro nascentes estudadas. Percebe-se que alguns indicadores nas áreas das nascentes periurbanas apresentam comportamento correspondente à sustentabilidade e/ou quase sustentabilidade, como, por exemplo, os indicadores de densidade habitacional, escolaridade, acesso a crédito, renda familiar, subindicadores de saneamento como coleta de resíduos, abastecimento de água, e os subindicadores de qualidade de água como cor, amônia e DBO, vazão (Cacimba da Rosa) e pluviosidade. Entretanto, esse desempenho dos indicadores não conseguiu alavancar um melhor ISH.

Gráfico 2- Nível de sustentabilidade hidroambiental das áreas de nascentes do rio Gramame

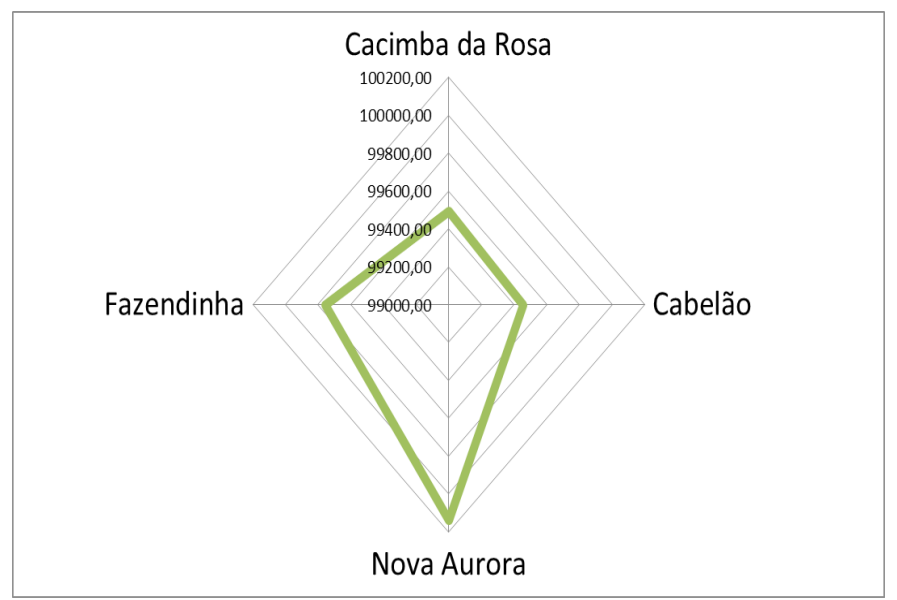

Fonte: Elaboração dos autores

\section{CONCLUSÃO}

Aavaliação da sustentabilidade hidroambiental das áreas estudadas é um processo complexo e multidimensional, visto a gama de informações e abrangência de variáveis. As propostas metodológicas de Calório (1997) e Daniel (2001) para áreas de nascentes são uma inovação e necessitam validar os resultados com a reaplicação do método por outras pesquisas científicas para que, dessa forma, possam ser adotadas como ferramentas de mensuração da sustentabilidade para os órgãos de controle ambiental da Paraíba e outros estados da Federação.

O sistema de indicadores utilizado neste estudo deve embasar as tomadas de decisões para melhorar a sustentabilidade hidroambiental das nascentes e ser reaplicado para acompanhamento do seu desempenho. Além disso, entende-se que a metodologia utilizada nesta pesquisa, deve ser testada para medir o nível de sustentabilidade hidroambiental em outras áreas 
de nascentes como também, em comunidades que praticam agricultura familiar.

Apesar das dificuldades de se colecionar dados e agrupar as informações de variáveis das quatro dimensões estudadas, pode-se inferir que os resultados são satisfatórios e representam a realidade local e devem ser utilizados para a gestão das áreas em torno das nascentes pesquisadas.

\section{AGRADECIMENTOS}

À Coordenação de Aperfeiçoamento de Pessoal de Nível Superior - Capes pela bolsa acadêmica concedida ao primeiro autor. Ao Conselho Nacional de Desenvolvimento Científico e Tecnológico $\mathrm{CNPq}$ pelo apoio financeiro concedido ao projeto de pesquisa "Análise do potencial de ações integradas para a restauração das funções ecológicas nas nascentes do rio Gramame (PB), sob a influência de unidades rurais de produção familiar".

\section{REFERÊNCIAS}

BALMFORD, A.; CRANE, P.; DOBSON, A. et al. The 2010 challenge: data availability, information needs and extraterrestrial insights. Phil Trans R Soc B, 2005, 360:221-228. DOI: 10.1098/rstb.2004.1599

BELLEN, H. M. van. Indicadores de Sustentabilidade: uma análise comparativa. 2. ed. Rio de Janeiro: Editora FGV, 2006.

BRASIL, Ministério do Meio Ambiente, Conselho Nacional do Meio Ambiente (Conama). Resolução Conama nº 357, de 17 de março de 2005. Brasília, DF: Diário Oficial da União, 2005.

BRUINS, H. J. et al. Degradation of Headwaters in the Arava Valley: anthropogenic and Climatic factors. Land Degradation \& Development. v.23. p.365-383, 2012.

CABRAL DA SILVA, T. et al. Avaliação de captações de águas de nascentes na bacia do rio Gramame. In: XIX Simpósio Brasileiro de Recursos Hídricos. Anais... Maceió/AL, 2011.

CALLADO, A. L. C. Modelo de Mensuração de
Sustentabilidade Empresarial: Uma Aplicação em Vinícolas Localizadas na Serra Gaúcha. Tese de Doutorado. Programa de Pós-Graduação em Agronegócios. UFRGS. Porto Alegre-RS, 2010.

CARÓLIO, M. C. Análise da sustentabilidade em estabelecimentos agrícolas familiares no Vale do Guaporé/MT. Dissertação (Mestrado) Programa de Pós-Graduação em Agricultura Familiar - Faculdade de Agricultura e Medicina Veterinária da Universidade Federal de Mato Grosso. Cuiabá.

CARVALHO, J. R. M. de; CURI, W. F. Construção de um índice de sustentabilidade hidro-ambiental através da análise multicritério: estudo em municípios paraibanos. 2013, Sociedade \& Natureza, 25(1), 91-105.

CASTEllO, L.; McGRATH, D. G.; BECK, P. S. Resource sustainability in small-scale fisheries in the Lower Amazon floodplains. Fisheries Research, 2011, 110(2), 356-364. DOI: 10.1016/j.fishres.2011.05.002

COELHO, V. H. R.; ALMEIDA, C. das N.; SILANS, A. M. B. P. de. Análise da Flutuação do Nível D'água para Estimativa da Recarga Subterrânea da Bacia Representativa do Rio Gramame no Nordeste Brasileiro. Revista Brasileira de Recursos Hídricos. v.17 n.2, Abr./ Jun. 2012, pp.241-252.

DANIEL, O.; COUTO, L.; SILVA, E.; GARCIA, R.; JUNCKSCH, I.; PASSOS, C. A. M. Alternativa a um método para determinação de um índice de Sustentabilidade. Viçosa/MG: Revista Árvore, v.25, n.4, p.455-462, 2001.

EEA(European EnvironmentAgency). Progress towards the European 2010 biodiversity target. EEA Report 4, 2009. Copenhagen, pp 1-52.

FELD, C. K., SOUSA, J. P., SILVA, P. M.; DAWSON, T. P. Indicators for biodiversity and ecosystem services: towards an improved framework for ecosystems assessment. Biodiversity and Conservation, 2010, 19(10), 2895-2919. DOI: 10.1007/s10531-010-9875-0

FELD, C. K.; BELLO, F. de; BUGTER, R., GRANDIN, U.; HERING, D.; LAVOREL, S.,; MOUNTFORD, O.; 
PARDO, I.; ... K. BRUCE, J. Assessing and monitoring ecosystems - indicators, concepts and their linkage to biodiversity and ecosystem services. D4.1 Review paper on ecological indicators. European Commission, 2007, 108pp. (UNSPECIFIED).

FELD, C. K.; MARTINS da SILVA, P.; PAULO SOUSA, J., de BELLO, F., BUGTER, R., GRANDIN, U.; ... HARRISON, P. Indicators of biodiversity and ecosystem services: a synthesis across ecosystems and spatial scales. Oikos, 2009, 118(12), 1862-1871. DOI: 10.1111/j.16000706.2009.17860.x

FERREIRA, M. J.; PEREIRA, I. M.; BOTELHO, S. A.; MELLO, C. R. de. Avaliação da regeneração natural em nascentes perturbadas no município de Lavras, MG. Santa Maria/RS: Ciência Florestal, v. 19, n. 2, p. 109129, abr.-jun., 2009.

FERREIRA, R. A.; AGUIAR NETTO, A. de O., SANTOS, T. I. S., SANTOS, B. L.; MATOS, E. L. de. Nascentes da sub-bacia hidrográfica do rio Poxim, estado de Sergipe: da degradação à restauração. Viçosa/MG: Revista Árvore, v. 35, n.2, p.265-277, 2011. DOI: http:// dx.doi.org/10.1590/S0100-67622011000200011

FILGUEIRA, H. J. A. (Coord.). Análise do potencial de ações integradas para a restauração das funções ecológicas nas nascentes do rio Gramame (PB), sob a influência de unidades rurais de produção familiar. Relatório técnico final. João Pessoa, PB: UFPB-CNPq, 2012. (Edital MCT/CNPq/CT-AGRONEGÓCIO/CTHIDRO - № 027/2008 - Projeto de Pesquisa). APHA/ AWWA/WEF. Standard methods for the examination of water and wastewater. $22^{\text {th }}$ edition. Public Health Association. New York, 2012.

FORTINI, L. B.; RABELO, F. G.; ZARIN, D. J. Mixed potential for sustainable forest use in the tidal floodplain of the Amazon River. Forest Ecology and Management, 2006, 231(1-3), 78-85. DOI: 10.1016/j. foreco.2006.04.038

FRY, L. M. Climate change and development impacts on the sustainability of Headwater-fed water supply systems in the Alto Beni region of Bolivia. Journal of Hydrology.
$468-469$ (2012) 120-129.

Guimarães, L. T. Proposta de um Sistema de Indicadores de Desenvolvimento Sustentável para Bacias Hidrográficas. Tese de Doutorado. Programa de Pós-graduação de Engenharia da Universidade Federal do Rio de Janeiro, Rio de Janeiro/RJ, 2008.

HAMMOND, A.;ADRIAANSE, A.; RODENBURG, E. et al. Environmental Indicators: a Systematic Approach to Measuring and Reporting on Environmental Policy Performance in the Context of Sustainable Development. Washington: WRI, 1995. 53p.

INSTITUTO BRASILEIRO DE GEOGRAFIA E ESTATÍSTICA(IBGE). Base de informações do Censo Demográfico 2010: resultados do universo por setor censitário. Documentação do Arquivo. Rio de Janeiro: IBGE, 2011.

JUNK, W.J. The central Amazon River floodplain: concepts for the sustainable use of its resources. pp. 75-94 In: JUNK W. J.; OHLY, J.J.; PIEDADE, M.T.F.; SOARES, M.G.M. (Eds.) The central Amazon floodplain: actual use and options for a sustainable management. Backhuys Publishers, Lieben.

KOBIYAMA M.; MOTA, A. A.; CORSEUIL, C.W. Recursos hídricos e saneamento. Curitiba: Ed. Organic Trading, 160p. 2008. VIEIRA, V. P. P. B. Recursos hídricos e o desenvolvimento sustentável do Semiárido nordestino. Revista Brasileira de Recursos Hídricos. Volume 1. n. 1. Jan/Jun, 1996, p. 89-107.

KOPERSKI, P., DUMNICKA, E., GALAS, J. Abiotic parameters determining fauna composition in karstic Headwaters. Polish Journal of Ecology. 59, 1: 153-163, 2011. (Regular research paper).

LINDENMAYER, D. B., MARGULES, C.R.; BOTKIN, D. B. Indicators of Biodiversity for Ecologically Sustainable Forest Management. Conservation Biology, 2000, 14(4), 941-950. DOI: 10.1046/j.15231739.2000.98533.x

MAGALHÃES JÚNIOR, A. P. Indicadores Ambientais 
e Recursos Hídricos: Realidade e Perspectivas para o Brasil a partir da Experiência Francesa. 2. ed. Rio de Janeiro: Bertrand Brasil, 2010.

MARTINS, M. F.; CÂNDIDO, G. A. Índice de Desenvolvimento Sustentável para Municípios (IDSM): metodologia para análise e cálculo do IDSM e classificação dos níveis de sustentabilidade - uma aplicação no Estado da Paraíba. João Pessoa: Sebrae/ PB, 2008.

MENEZES, M. D. de; JUNQUEIRA JÚNIOR, A. J.; MELLO, C. R. de, SILVA, A. M. da, CURI, N.; MARQUES, J. J. Dinâmica hidrológica de duas nascentes, associada ao uso do solo, características pedológicas e atributos físico-hídricos na sub-bacia hidrográfica do Ribeirão Lavrinha - Serra da Mantiqueira (MG). Scientia Florestalis, Piracicaba, v. 37, n. 82, p. 175-184, jun. 2009.

MORA, M. A.; GRANT, W. E.; WILKINS, L.; WANG, Hsiao-Hsuan. Simulated effects of reduced Headwater flow from the Edwards Aquifer on population size of the fountain darter (Etheostoma fonticola). Ecological Modelling, 250: 235-243, 2013. DOI: 10.1016/j. ecolmodel.2012.11.013

OECD. Organization for Economic Cooperation and Development. Core set of indicators for evironmental performance reviews. Paris: OECD, 1994.

PARAÍBA. Governo do Estado da Paraíba, Secretaria Extraordinária do Meio Ambiente, dos Recursos Hídricos e Minerais. Plano diretor de recursos hídricos da bacia hidrográfica do rio Gramame. v. 1. João Pessoa, PB: Secretaria Extraordinária do Meio Ambiente, dos Recursos Hídricos e Minerais, 2000. 247p. (Relatório Final).

PROGRAMA DAS NAÇÕES UNIDAS PARA O DESENVOLVIMENTO (PNUD). Relatório do Desenvolvimento Humano-RDH. 2006. Disponível em: <http://www.pnud.org.br.rdh> Acesso em: $22 \mathrm{de}$ nov 2012.

SANTOS, M. J. dos. Programa um milhão de cisternas rurais: proposição de um sistema de indicadores de avaliação de sustentabilidade SIAVS-P1MC. Tese (Doutorado). 2010, 242f. Universidade Federal de Campina Grande. Centro de Tecnologia e Recursos Naturais. Pós-Graduação e Recursos Naturais. Campina Grande, 2010.

SHAHIN A.; MAHBOD M. A. Prioritization of key performance indicators: an integration of analytical hierarchy process and goal setting. Int J Prod Perform Manage, 2007, 56:226-240. DOI: http://dx.doi. org/10.1108/17410400710731437

SINGH, R. K., MURTY, H. R., GUPTA, S. K.; DIKSHIT, K. An overview of sustainability assessment methodologies. Ecological Indicators, 2012, 15(1), 281-299. DOI: 10.1016/j.ecolind.2011.01.007

SINGH, R. K., MURTY, H. R., GUPTA, S. K.; DIKSHIT, K. An overview of sustainability assessment methodologies. Ecological Indicators, 2009, 9(2), 189-212. DOI: 10.1016/j.ecolind.2008.05.011

SOARES, J. C. de O.; SOUZA, C.A. de; PIERANGELI, M. A. Nascentes da sub-bacia hidrográfica do córrego Caeté/MT: estudo do uso, topografia e solo como subsídio para gestão. Taubaté/SP: Revista Brasileira de Gestão e Desenvolvimento Regional. v. 6, n. 1, p. 22-51, jan-abr/2010.

SWART, R., et al. Critical challenges for sustainability science. Science, 297, 2002, p.1994-1995.

VIEIRA, P. M. S.; STUDART, T. M. C. Proposta Metodológica para o Desenvolvimento de um Índice de Sustentabilidade Hidroambiental de Áreas Serranas no Semiárido Brasileiro - Estudo de Caso: Maciço de Baturité, Ceará. Revista Brasileira de Recursos Hídricos. Volume 14 n.4 Out/Dez, 2009, 125-136.

VIEIRA, V.P.P.B. Recursos hídricos e o desenvolvimento sustentável do Semiárido nordestino. Revista Brasileira de Recursos Hídricos. Volume 1. n. 1. Jan/Jun, 1996, p. 89-107.

$\mathrm{Xu}, \mathrm{Fu}$-Liu. A triangle model for evaluating the sustainability status and trends of economic development. Ecological Modeling. 195, 327-337, 2006. DOI: 10.1016/j.ecolmodel.2005.11.023 
ERRATA 02:

No artigo $<$ SUSTENTABILIDADE HIDROAMBIENTAL DE NASCENTES NA BACIA HIDROGRÁFICA DO RIO GRAMAME NO ESTADO DA PARAIIBA, BRASIL >, com número de DOI: < http://dx.doi. org/10.1590/1982-451320150307>, publicado no periódico $<$ Revista Sociedade\&Natureza $>,<$ vol. $27>(<$ num. 3>): $<453-468>$, na $<$ pagina $453>$ onde se lia:'< Hamilcar José Almeida Figueira $>$ 'leia-se:'< Hamilcar José Almeida Filgueira $>^{\prime}$

DOI: http://dx.doi.org/10.1590/1982-451320160114 